\title{
TOWARDS A SEMICLASSICAL SEISMOLOGY OF BLACK HOLES*
}

\author{
Michael Crescimanno \\ Center for Theoretical Physics \\ Laboratory for Nuclear Science \\ and Department of Physics \\ Massachusetts Institute of Technology \\ Cambridge, Massachusetts 02139 U.S.A.
}

Submitted to: Phys. Rev. D (D.O.E.) under contract \#DE-AC02-76ER03069, and by the Division of Applied Mathematics of the U.S. Department of Energy under contract \#DE-FG02-88ER25066. 


\begin{abstract}
Black hole spacetimes are semiclassically not static. For black holes whose lifetime is larger than the age of the universe we compute, in leading order, the power spectrum of deviations of the electromagnetic charge from it's average value, zero. Semiclassically the metric itself has a statistical interpretation and we compute a lowerbound on its variance.
\end{abstract}




\section{INTRODUCTION}

Since the seventies after the remarkable work of Hawking ${ }^{1}$, Fulling ${ }^{2}$ and others, there has been a flowering of technical development and interest in semiclassical gravity*. The central problems of this approach, already outlined in some of the very earliest papers, include understanding the meaning of static solutions, the apparent loss of unitary evolution in black hole spacetime, and the nature of the endpoint of black hole evaporation. It is difficult to ascertain whether these questions are addressable with semiclassical techniques, and many researchers have found it instructive to study these questions in low-dimensional models such as two-dimensional dilaton gravity ${ }^{3}$.

One of the central results of semiclassical gravity as it applies to Schwarzschild spacetimes is that the Green function of quantum fields is periodic in imaginary time. This means that one should regard the fields outside the hole as being excited and being characterized approximately in terms of a thermal distribution. An observer at rest in these co-ordinates but out in the asymptotically flat region will see a thermal flux of particles emerging from the hole. It is understood that this Hawking radiation is responsible for the decay of the black hole.

Of course, since the black hole has a finite total energy, it's dissolution into Hawking radiation is not a smooth process; in a fixed time interval the amount of energy it radiates varies statistically. The semiclassical result represents the time averaged rate of the process. Simply put, the Hawking process is "noisy". Unfortunately, the evaporation process is, by nature, a non-equilibrium process and would be very difficult even in principle to model ex-

\footnotetext{
* By semiclassical gravity we mean treating matter fields quantum mechanically in classical geometrical backgrounds.
} 
actly. In order to avoid non-equilibrium questions we rigorously answer what we feel are physically interesting questions about equilibrium states entirely within the context of semiclassical gravity. Since for most of its evolution the black hole spacetime is well approximated as a quasi-equilibrium state, assuming equilibrium seems physically reasonable. Indeed, the static classical behavior of four-dimensional black holes in equilibrium with a radiation bath is a well studied problem ${ }^{4}$. Furthermore, experience with many dissipative systems even far from equilibrium indicates that often the actual noise has nearly the same norm and statistical properties as noise computed assuming equilibrium conditions.

This work describes a computation of the power spectrum of the charge of a fourdimensional black hole assuming equilibrium. We do this in a regime where the semiclassical response to charging the hole is given by a linear equation, although this analysis may be extended to the non-linear regime. The results we obtain yield a lower limit on the the statistical variations of the metric itself, which is interpretable as the "seismology" of the spacetime's geometry. We treat the metric as a background field and so seismology arises in a different way than that of Ref.[5] where the gravitational field is treated dynamically and semiclassically. Section II contains an introduction to equilibrium notions and noise, Section III is a computation of the power spectrum of the charge for a black hole and Section IV discusses the physical effects and back reaction on the metric.

\section{CHARGE AND EQUILIBRIUM}

In this paper we will restrict our attention to nearly static solutions of Einstein's equations coupled semiclassically to the usual electromagnetic field and electron. Thus we are interested 
in nearly static solutions that extremize the action

$$
S=\int d^{4} x \sqrt{g}\left[R+\frac{1}{4} F^{2}+i \bar{\psi}(\not D-m) \psi\right]
$$

We will show below that the results will not depend on the particular type of charged matter field that one uses, and only on the total number, $N$, of "electron" (lowest mass, charged) species. For the purpose of simplicity here it we require that all the lowest mass charged species have the same charge. Below, when discussing numbers we use the physical (spin) degeneracy $N=2$ but it is useful for reference to retain the $N$-dependence explicitly in some of the formulae.

The static electrical properties of black holes are summarized by the well known ReisnerNordstrom (RN) solution to Eq.(2.1), whose metric and gauge field are (in the usual spherical co-ordinates $t, r, \theta, \phi$,

$$
\begin{gathered}
g_{\mu \nu}=\operatorname{diag}\left(B,-1 / B,-r^{2},-r^{2} \sin ^{2} \theta\right), \\
A_{\mu}=(-Q / r, 0,0,0)
\end{gathered}
$$

where $B=1-2 M / r+Q^{2} / r^{2}$, and with $M$ and $Q$ being the mass and charge of the hole, respectively. The metric has two horizons, $r_{ \pm}=M \pm \sqrt{M^{2}-Q^{2}}$, and semiclassically the temperature of the outgoing radiation as seen from a stationary Schwarzschild observer at infinity is $1 / \beta=\frac{r_{+}-r_{-}}{4 \pi r_{+}^{2}}$.

The semiclassical evolution of the RN solution has been studied extensively. One of the earliest references is due to Gibbons ${ }^{6}$ where he computed the charge loss rate, mostly with an interest in understanding the super-radiative regime where $m M<e Q$ with $m$ and $e$ being the mass and charge of the electron, the lightest charged species. This work was followed up by many other authors ${ }^{7,8,9,10}$, which studied in more generality and precision the evaporation of 
charged black holes into empty space. These works all use the simplest result of the Hawking calculation, namely expressions for the time-averaged rate of energy or charge loss, and the conclusions of these earlier works are summarized phenomenologically in terms of decay rates for the $M$ and $Q$ parameters of Eq.(2.1). Thus, from the point of view of this simple use of semiclassical gravity, one finds that the charge always dissipates away, eventually leaving a neutral hole. The main point of this paper will be to show in a quantitative way that this expectation is too naive. Instead, if we regard even an initially neutral hole as a quasiequilibrium state, the charge of that hole will fluctuate about zero. Let $<>$ represent an average over the equilibrium ensemble. Although charge symmetry for the spacetime of a neutral black hole suggests that $\langle Q\rangle=0$, the charge on the hole will undergo statistical fluctuations so that $<Q^{2}>\neq 0$. This fact was known long ago ${ }^{8,11}$, and may be understood as a simple consequence of the equipartition theorem. Since the energy in the electrostatic field outside of a hole of mass $M$ and charge $Q<<M$ is

$$
U=\frac{Q^{2}}{2 r_{+}}
$$

we expect in equilibrium that this energy will be $\sim \frac{1}{2 \beta}$ so that

$$
<Q^{2}>\sim \frac{1}{4 \pi \alpha}
$$

in electron units. Note that this result is independent of the mass of the black hole, and also independent of the mass of the electron and the number of electron species.

It is important to emphasize that this $\left\langle Q^{2}>\right.$ is not a consequence of the granularity of charge, and is to be understood as a statement about an ensemble of identically prepared black holes (or equivalently, one black hole observed over a long time) in equilibrium with 
a heat bath. Thus, it is no difficulty that $\sqrt{\left\langle Q^{2}>\right.} \neq \mathbf{Z}$ in electron units. This result also holds for astrophysical black holes in the limit of low total charge. Of course, in most practical applications, an astrophysical black hole in a space plasma will tend to acquire a net positive charge (which would be, in practice, much bigger than that implied by Eq.(2.4)) as a consequence of the well known Langmuir effect ${ }^{12}$, which has nothing to do with the effects of semiclassical gravity. We restrict our study to that of a black hole in either empty space or a box filled with thermal radiation comprised solely of photons, electrons and positrons but in thermal equilibrium with the hole.

\section{EQUILIBRIUM CORRELATION FUNCTIONS}

The results of the previous section indicate that the average charge of a nearly neutral black hole at equilibrium is zero $(<Q>=0)$ but that the average charge-squared $<Q^{2}>$ is non-zero. If one throws charge into a neutral black hole of macroscopic horizon radius it will stay charged for a very long time, and so the above result, although correct, needs further elucidation. In particular, it would be of most interest to calculate the charge-charge correlation function $<Q(t) Q(0)>$. In this section we compute this, and indeed semiclassically all correlation functions of the charge of a black hole in equilibrium but only in the limit that the total charge on the hole is small. By low charge we mean the ratio, $\Lambda$, of electromagnetic force and gravitational force on an electron is less than one; $\Lambda=e Q / m M<1$. Note that since $e / m>>1$ in rationalized units, the Hawking temperature for the RN spacetime (which is given above in terms of $M$ and $Q$ ) is essentially independent of the charge.

In computing this correlation function we will re-derive and use a generalization of the fluctuation-dissipation theorem $(\mathrm{FDT})^{13}$ for curved space. This theorem will ultimately relate 
the charge-charge correlator ('fluctuation') to a 'linear transport coefficient' that characterizes the evaporation of the black hole's charge. The linear transport coefficient for charge loss follows directly from the results of Hawking, Fulling, Gibbons and others using the quasiequilibrium notions of semiclassical gravity. It has long been known that the best description of a black hole spacetime semiclassically in equilibrium is as an ensemble of spacetimes 'nearby' a given solution ${ }^{11}$. Also, the variance of this ensemble was computed by various means ${ }^{11,14,15}$. Page $^{8}$ computed the likelyhood of any particular measurement of the charge of a black hole, essentially finding a gaussian probability with variance given by Eq.(2.4).

One way to understand the results we present here is that we will compute more than just these 'static' (i.e. equal-time) variances such as Eq.(2.4). Instead of computing averages over the ensemble (simple phase averages) we use a version of the FDT to compute properties about the average temporal evolution of a single system in the ensemble. Noise (that is, correlation functions) in a co-ordinate of a sub-system can be thought of as an average over an ensemble of trajectories in the ensemble used to compute phase averages. The temporal evolution of the sub-system is a consequence of the fact that it is dissipatively coupled to the rest of the system.

It is very natural to understand the Hawking effect as a dissipative phenomena. Dissipation is generic to situation in which one studies a particular degree of freedom (for example, the metric or the charge of the hole) of a system that is coupled to a continuum of other degrees of freedom (here quantum fields) whose precise evolution is treated approximately. This connection between the Hawking effect and dissipation has been explored mostly in the context of isotropic cosmological models ${ }^{16,17}$.

Strictly speaking, for the FDT we develop and use here, we are formally assuming that 
the charge is a continuous variable and so are implicitly working in the approximation that $\alpha$ is small. Versions of the FDT has been used before in semiclassical general relativity for the back-reaction on the gravitational shear due to the emission of gravity waves ${ }^{18}$ and studying the stability of AdS spacetimes ${ }^{19}$ but we use it in a rather different way here.

To begin with, consider that the number of charged quanta of spin $s$ (in the charged matter field of Eq.(2.1), $s=1 / 2$ ) emitted by an isolated RN black hole (Eq.(2.2)) per unit time per unit volume of final state phase space as seen by an asymptotic Schwarzschild observer is

$$
\frac{d n^{ \pm}}{d t}=\frac{N \sigma_{E}^{ \pm}}{e^{\beta\left(E \pm e \Phi_{h}\right)}+(-1)^{2 s+1}},
$$

where $e$ is the charge of the matter field. $\sigma_{E}^{ \pm}$is simply the total absorption cross-section. The energy, $E$, is $\sqrt{\mathbf{p}^{2}+m^{2}}$ and $\Phi_{h}=Q / r_{+}$is the 'voltage' of the horizon w.r.t. infinity. Gibbons ${ }^{6}$ computed the rate of loss of charge from a black hole by estimating $\sigma_{E}^{ \pm}$approximately via the wave equation in the RN background. His work was later extended and made more precise by Ternov, et. al. ${ }^{7}$. In the $\Lambda<1$ regime it is especially easy to obtain their results with a classical computation of $\sigma_{E}^{ \pm}$and since this will suffice in what follows we briefly describe it's derivation. Approximately, the total absorption cross-section $\sigma_{E}^{ \pm}$is

$$
\sigma_{E}^{ \pm}=\frac{A^{ \pm}}{E^{2}-m^{2}}
$$

where $A^{ \pm}$is found by solving the geodesic equation and to lowest non-vanishing order in $M$ and $Q$ is

$$
A^{ \pm}=4 \pi M^{2} m^{2}(1 \mp \Lambda)+\ldots
$$

Now, using the fact that the total current at infinity as given in terms of $d n^{ \pm} / d t$ is

$$
\dot{Q}=e \int\left(\frac{d n^{+}}{d t}-\frac{d n^{-}}{d t}\right) d^{3} \mathbf{p}
$$


and furthermore, for holes whose lifetimes are long compared with the present age of the universe and using $m>1 / \beta$

$$
\frac{1}{e^{\beta\left(E \pm e \Phi_{h}\right)} \pm 1} \sim e^{-\beta\left(E \pm e \Phi_{h}\right)}
$$

and working in the $\Lambda<1$ regime we can use Eq.(3.2-3.5) to relate the net current at infinity to the hole charge

$$
\dot{Q}=-Q / \tau
$$

where

$$
\tau=\frac{\lambda^{2}}{8 \pi \alpha c r_{h} N} e^{4 \pi \frac{r_{h}}{\lambda}}
$$

is the lifetime of charge on the hole in terms of $\lambda$, the compton wavelength of the lightest charged particle, and $r_{h}(\sim 2 M)$, the Schwarzschild radius of the hole ( $c$ is the speed of light.) Notice that the charge loss is proportional to the total charge and $\tau$ is only a function of $m$, $M$, the Planck scale and $\alpha$. Note that this decay time $\tau$ of Eq.(3.7) is of a very different from that found in the $\Lambda>>1$ limit (compare with Ref.[6,7,9].) This is due to the fact that in the $\Lambda<1$ regime we ignore the small contribution to the rate formula due to "super-radiance" that arises from pair creation in the constant electromagnetic field ${ }^{19}$.

To an observer in Schwarzschild co-ordinates, the evaporation of the charge of the hole has a very simple local explanation. Such an observer would conclude that since the quasiequilibrium state corresponds to putting the black hole in a box filled with radiation at the temperature of the hole, the loss of charge from the hole is simply a result of the charges in the bath neutralizing the hole. Indeed, $\tau$ has the dimensions of electrical resistivity and it is precisely the resistance to the flow of the electron and positrons in the geometrical background of the hole. It is instructive to make this interpretation quantitative. Note that the resistivity, 
$\rho$, (supposing the metric is approximately a flat Minkowski metric) of a thermal bath is given by $1 / \rho \sim n_{e} e \frac{\partial V}{\partial \mathbf{E}}$ where $n_{e}$ is the number density of electrons and positrons in the bath

$$
n_{e} \sim N\left(\frac{m}{\beta}\right)^{3 / 2} \exp (-\beta m)
$$

and $\frac{\partial V}{\partial \mathbf{E}}$ is the drift velocity per unit applied electric field. This may be easily estimated by $\frac{\partial V}{\partial \mathbf{E}}=e l / m V$ where $V$ is the mean velocity of the electrons in the bath $\left(\sim \beta^{-1 / 2}\right)$ and $l$ is a length scale for scattering. In the low temperature limit the scattering due to the interaction of the drifting charges with the other particles in the bath is negligible, and $l$ is well estimated by a gravitational scattering length. The gravitational scattering length is the length over which a thermal particle in the vicinity of the hole would experience an $\mathcal{O}(1)$ change in its velocity, $l \sim G_{N} M m \beta \sim M^{2} m$ Putting this all together, we conclude

$$
\rho \sim \frac{e^{\beta m}}{\alpha m^{2} M N}
$$

which has the same functional dependence as the analytical result Eq.(3.7).

We now outline the general argument due to Callen and Welton ${ }^{13}$ as used by Kubo ${ }^{21}$ and generalized by Martin and Schwinger ${ }^{22}$ that relates the dissipation described in Eq.(3.7) to the equilibrium fluctuations in the charge-charge correlation function. On general grounds in the interaction picture we may write the response (current) to the presence of a gauge field $A^{\nu}$ to first order in terms of the commutator of two currents,

$$
<J_{a}>=<J_{a}>_{0}+\int<\left[J_{a}, J_{\nu}\right]>A^{\nu}+\ldots
$$

where $\left\langle J_{a}>_{0}\right.$ is the current when there is no applied electric field (by charge invariance of our neutral black hole state we ascertain that $\left\langle J_{a}\right\rangle_{0}$ is zero) and where roman indices refer 
only to space components (well defined by the existence of a global time-like killing vector in the spacetime of Eq.(2.2)) whereas greek indices are Minkowski.

It is convenient to define the local conductivity tensor $\sigma_{a}^{b}(\omega)$ that relates the current $<J_{a}>$ to the total electric field $E_{b}=\int E_{b}(\omega) e^{-i \omega t} d \omega$

$$
<J_{a}(\omega)>=\sigma_{a}^{b}(\omega) E_{b}(\omega)
$$

and by standard manipulations of Eq.(3.10), computing as a local Schwarzschild observer would, we find the Kubo ${ }^{21}$ result,

$$
\sigma_{a}^{b}(\omega)=\frac{1}{\omega} \int_{0}^{\infty} d t e^{i \omega t}<\left[J_{a}(t), J^{b}(0)\right]>+i \frac{\Gamma}{\omega} \delta_{a}^{b}
$$

with $J_{a}$ being the current averaged over space and where $\Gamma$ is an infinite constant associated with the spectral density of the photon propagator. The term involving $\Gamma$ is a trivial consequence of the fact that, by gauge invariance, the equal time commutator of a time and a space component of the electromagnetic current cannot be zero. It is essentially the polarizability of the vacuum modes, and of course cannot lead to any dissipative effects ${ }^{23}$. Now, although we might guess that the commutator of two space components of the current is also zero, in view of Eq.(3.6) this can clearly not be the case in the curved space of Eq.(2.2). We are interested in the part of the conductivity $\sigma_{a}^{b}$ that is even in frequency $\omega$. By trivial manipulations of Eq.(3.12) we have

$$
\sigma_{a}^{b}(\omega)+\sigma_{a}^{b}(-\omega)=\frac{1}{\omega}<\left[J_{a}, J^{b}\right]>(\omega)
$$

As shown in Ref.[24], the correlation functions of fields in a black hole spacetime behave asymptotically as finite temperature Green functions at the temperature of the hole. Thus, they satisfy the well-known KMS condition and use of that condition allows one to relate the 
fourier components of the commutator and product of two currents,

$$
<\left[J_{a}, J^{b}\right]>=(\exp (\beta \omega)-1)<J_{a} J^{b}>
$$

The FDT in this context is just Eq.(3.13), the dissipation, taken together with Eq.(3.14), $<J_{a} J^{b}>$ representing the fluctuation*. Thus integrating the radial current in Eq.(3.6) that arises as a response to the electric field (caused by the charge on the hole) over a two sphere and putting all the metric factors in Eq.(3.13), the high temperature limit $(\beta \rightarrow 0)$ of Eq.(3.14) becomes,

$$
<Q_{\omega} Q_{-\omega}>\sim \frac{2}{\beta R \omega^{2}}
$$

where $R=\tau / r_{+}$has dimensions of resistance. Notice that this result diverges as $\omega \rightarrow 0$. Since Parsifal's identity,

$$
<Q^{2}>=\frac{1}{2 \pi} \int<Q_{\omega} Q_{-\omega}>d \omega
$$

for Eq.(3.15) implies that $<Q^{2}>$ is infinite which one knows (see Eq.(2.4)) cannot be correct. Indeed, in the exposition above we have neglected the back reaction of the charge loss on the subsequent evolution of the hole's charge. This may be seen most directly by looking at the correlator as a function of time. Fourier transforming Eq.(3.15) we find the correlation function for brownian motion,

$$
<Q(t) Q(0)>=|t| / \beta R
$$

This would imply that if you started with a neutral hole that after some time the charge would have drifted quite far from zero. This is what we would expect perhaps for the evolution of a

* As described later, for $\Lambda<1$ we will be most interested in the high-temperature limit of the FDT and so the Wightman function (RHS of Eq.(3.14)) will well approximate the causal Green function that one would detect experimentally. 
global charge of the hole but not for a charge associated with a gauged symmetry. Indeed, this Brownian-like behavior of the charge tells us that we have unwittingly neglected the biasing of the charge loss by the electrostatic energy of the charge of the hole!

Thus far we have neglected the $F^{2}$ term in the Lagrangian Eq.(2.1), and it is easy to see that including it in the analysis will modify the small $\omega$ behavior of Eq.(3.15). The correct formula for the charge-charge correlators may be found by exploiting an electrical analogy. From Eq.(2.3) above and from the charge decay formula Eq.(3.6), it is natural to think of a nearly neutral black hole as a circuit comprised of a capacitor (of value $r_{+}$, the hole 'radius') shunted by a resistor (of resistance $R=\tau / r_{+}$). Here the resistor is materially caused by the quantum fields outside the hole and thus is in a quasi-equilibrium state at temperature $1 / \beta$. It is a well know consequence of the FDT that a resistor at a high temperature/low frequency acts as a source of 'white' voltage noise,

$$
<V_{\omega} V_{-\omega}>=R / \beta
$$

and so the equivalent circuit diagram of our nearly neutral black hole is as shown in figure 1 . Using Kirchoff's laws it is now simple to ascertain the charge-charge correlator

$$
<Q_{\omega} Q_{-\omega}>=\frac{R r_{+}^{2}}{\beta\left(1+\omega^{2} \tau^{2}\right)} .
$$

This formula is finite as $\omega \rightarrow 0$ and as expected yields Eq.(2.4) when integrated via Eq.(3.16)*. Also, for a linear system, such as the loss of charge off of a nearly neutral hole for $\Lambda<1$, all

* Actually, Eq.(3.19) is the high temperature limit of the full FDT result

$$
<Q_{\omega} Q_{-\omega}>=\frac{R r_{+}^{2} \omega}{\left(1+\omega^{2} \tau^{2}\right)(\exp (\beta \omega)-1)} .
$$

Most of the support for this correlation function comes from the region $\omega<1 / \tau$ and since for $\Lambda<1, \beta / \tau \sim 0$, Eq.(3.19) is a very good approximation to the full FDT result. 
higher correlation functions of the charge descend from the two-point correlator in the usual way. This result generalizes the equal-time correlators of Page ${ }^{8}$.

\section{CONSEQUENCES AND A SEISMIC LOWER BOUND}

We now discuss a few consequences of the power spectrum found in the previous section for the charge of a nearly neutral black hole. We first discuss some issues about the classical phenomenology of this noise spectrum and finally discuss questions about its effect on the structure of the spacetime itself.

The correlation function Eq.(3.19) depends on a single time scale $\tau$. As seen from Eq.(3.7) this time scale can be truly huge for astrophysical black holes. In those cases, all the 'noise' of Eq.(3.19) happens at incredibly low frequencies and for all practical purposes we would say that the charge of the black hole was a constant. However, for a medium-sized hole whose lifetime is still long compared with the lifetime of the universe the noise can have support at 'everyday' frequencies. For example, for a black hole that would have a lifetime of a hundred times the age of the observable universe we find that $\tau \sim 10^{-6}$ seconds and by Eq.(3.19) that the hole charge is 'noisy' into megahertz frequency range.

What would be a practical observable consequences of this charge noise? It is interesting to calculate the effect this stochastic charge of the black hole would have on the scattering of a slowly (with respect to $r_{+} / \tau$ ) moving particle of fixed charge. One finds that the noise has a tendency to repel charges, regardless of their sign, away from the hole! This rather curious-sounding conclusion can be reached a variety of ways, perhaps most instructively by integrating over the Gaussian noise kernel Eq.(3.19) in the path integral for the test particle. The leading effect may be understood by paying particular attention to the zero-temperature 
limit (see the footnote below Eq.(3.19)), as one does in the related case of dissipative quantum mechanics ${ }^{25}$ when interested in the classical limit of the effective equations of motion. The path integral for a test particle of unit charge $( \pm)$ moving under the influence of a stochastic charge $Q$ is,

$$
Z=\int[d Q][d \mathbf{x}] \mathrm{M}(Q) \exp \int d t\left\{\frac{m}{2} \dot{\mathbf{x}}^{2} \pm \frac{Q}{|\mathbf{x}|}\right\}
$$

Where we have for simplicity ignored the gravitational attraction of the hole. $\mathrm{M}(Q)$ is the gaussian density whose variance is given by Eq.(3.19), in the low temperature limit. Performing the integration over $Q$ we find

$$
Z=\int[d \mathbf{x}] \exp \int d t\left\{\frac{m}{2} \dot{\mathbf{x}}^{2}+\frac{1}{2 R} \int d t^{\prime} \frac{1}{|\mathbf{x}(\mathbf{t})|} \theta\left(t-t^{\prime}\right) \frac{1}{\left|\mathbf{x}\left(\mathbf{t}^{\prime}\right)\right|}\right\}
$$

Writing down the equations of motion that follow from this lagrangian, we see that the last term contributes a term that is odd under $t \rightarrow-t$. Non-local terms ('memory') in equations of motion are typical of the low-temperature limit of dissipative systems (see for example Ref.[24].) The net effect of the fluctuations of the charge of the hole is to create an effective repulsive force for charged test particles of either sign! As expected, the smaller the hole, the larger repulsion is for slowly moving test charges. Furthermore, the repulsion is proportional to the square of the charge of the particle. This behavior may be understood qualitatively from very simple models and is borne out well quantitatively in computer simulations of the motions of test charges under the influence of a stochastic charge of this type.

From the point of view of the correlation function Eq.(3.19) and the charge loss equation Eq.(3.6) we may make a simple point about the classical version of the information loss paradox. The classical version of the information loss paradox in this context asks if it is possible to reproduce the initial time-dependent current that was used to charge a hole given 
only the resulting evaporation current. Indeed, Eq.(3.19) informs us that we should expect there to be all sorts of temporal correlations in the evaporation current that don't carry any 'information' about the initial charging current but that are a result of the quasi-equilibrium state. Furthermore, the first-order equation for the charge loss indicates that it will not be possible to simply 'evolve back' the fourier modes of the measured evaporation current. Thus, as expected in this semiclassical picture, it is not possible to recover the information about the time-dependence of the initial charging current: electrically black holes act as lossy low-pass filters. Of course, one hopes that understanding evaporation non-perturbatively will ameliorate both this classical and the quantum mechanical information loss problem.

Another interesting consequence of the stochastic nature of the charge of the black hole is that the metric inherits a statistical interpretation. The metric of Eq.(2.2) for a static charged black hole has $g_{00}=1-2 M / r+Q^{2} / r^{2}$, and since we expect $Q^{2}$ to vary in accordance with Eq.(3.19) we expect that as a consequence of back-reaction, the metric itself has statistical dispersion. Rather than solve the Vadia-Bonner metric for the time dependent metric exactly we approximate the true solution by the RN solution with time dependent $M$ and $Q$. Indeed, for a small hole in a box filled with thermal radiation, thermal equilibrium suggests $<\delta M>=$ 0 and we expect that the noise in the mass fluctuations has most of its support at lower frequencies* Thus, the total metrical dispersion, in the high frequency $(\omega>1 / \tau$ but $\omega<1 / \beta$, beyond which all noise is exponentially damped anyways.) limit, should be strictly larger

* We would expect this to be true whenever the timescale $\tau$ describing the charge loss is small compared with the lifetime of hole were it to radiate into an empty vacuum. This is precisely the case for a black hole whose lifetime is shorter than about $10^{4}$ times the present age of the universe. 
than that due to the charge-charge correlator Eq.(3.19)

$$
\begin{gathered}
g_{00}(\omega)=\int d t e^{i \omega t} g_{00}(t) \\
<g_{00}(\omega) g_{00}(-\omega)>>\frac{\tau}{4+\omega^{2} \tau^{2}} \frac{1}{r_{1}^{2} r_{2}^{2}}
\end{gathered}
$$

Seismology is the study of the temporal evolution of the geometry of quasi-static objects. Eq.(4.3) may be thought of as a lower bound for the seismic activity in the geometry of the spacetime of a black hole, as seen by a stationary asymptotic observer. If one interprets Eq.(4.3) and the correlator Eq.(3.19) as noise in the radial position of causal horizon, we find that the horizon dilates out and in several multiples of the hole's compton wavelength. These are very small 'quakes' but correspond to radial dilation that could be larger than the distance between the horizon and the stretched horizon and so may be of consequence

for understanding what an asymptotic observer sees happening at the stretched horizon ${ }^{26,27}$. Note that if we fix $\omega$ and pass to the thermodynamic limit $(M \rightarrow \infty)$ these fluctuation vanish, as expected on general principles. also, although Eq.(4.3) is second order in the temperature (that is, second order in Planck's constant) it is the leading term and going beyond one loop in the quantum fields will generate higher order terms. Also, these radial dilations are completely distinct from the dispersion in the position of $r=0$ due to momentum conservation during evaporation of Hawking quanta. They are also distinct from the dispersion of the gravitational shear of Ref.[18]. It is straightforward to compute the 'seismology' of the hole's geometry in higher spherical harmonics, but that is technically more complicated.

\section{LANGEVIN VIEW OF SEMICLASSICAL GRAVITY}

Of course not only the charge $Q$ but also, $M$, the mass of the hole evolves semiclassically, and to really compute the correlations of the metric response we need to all the correlators 
$<M M>,<M Q>,<Q Q>$ (we neglect angular momentum evolution to simplify the discussion) and then compute the linear response about the metric Eq.(2.2) using the Einstein equations. This is a Langevin-like approach to the back reaction in Einstein equations. Usually, in studying the back reaction semiclassically one solves

$$
G_{\mu \nu}=<T_{\mu \nu}>
$$

where $\left\langle T_{\mu \nu}>\right.$ is the renormalized stress tensor in which appropriate boundary conditions are taken to match with the appropriate physical situation, for example, the evaporation of the hole. Since the spacetimes one is most interested in often have finite asymptotic masses and charges, we have argued in this paper that it is important to take into account the fluctuations about these values. The fluctuations for a black hole radiating into empty space are likely to be very close to those computed assuming equilibrium. Thus, in this Langevin-like approach to semiclassical gravity we would replace Eq.(5.1) with Einstein's equation

$$
G_{\mu \nu}=T_{\mu \nu}
$$

and to solve this for the variables $g_{\mu \nu}$ and their statistical properties, we specify $<T_{\mu \nu}>$ and $<T_{\mu \nu}(x) T_{\alpha \beta}\left(x^{\prime}\right)>$ and all the corelation functions of the source $T$ assuming equilibrium. This is difficult to do in practice for technical reasons. In this paper we have not solved Eq.(5.2) but the technically simpler situation of Einstein-Maxwell where we have treated only the electromagnetic current and its back reaction on the metric as a Langevin system. Understanding semiclassical gravity from this quasi-equilibrium viewpoint which philosophically treats back reaction as a Langevin-like problem could lead to further elucidation of the properties of black holes. 


\section{Acknowledgements}

The author has profitted greatly from conversations with S. Axelrod, D. Freed, D. Freed-

man, G. W. Gibbons, R. Jackiw, E. Keski-Vakurri, A. Landsberg, S. Mathur, M. Ortiz, D. N. Page, M. Perry and I. M. Singer. The author wishes to thank J. W. York, Jr. for pointing out the connections between this work and Ref.[5]. 


\section{REFERENCES}

1 S. W. Hawking, Commun. Math. Phys. 43, 199 (1975).

2 S. A. Fulling, Phys. Rev. D7, 2850 (1973).

3 C. Callan, S. B. Giddings, J. A. Harvey and A. Strominger, Phys. Rev. D45, 1005 (1992), R. B. Mann and T. G. Steele, Class. Quant. Grav. 9, 475 (1992); for charged black hole solutions in 2-d see, M. D. McGuigan, C. R. Nappi, and S. Yost, Nucl. Phys. B375, 421 (1992), G. W. Gibbons and M. J. Perry, Int. J. Mod. Phys. D1, 335 (1992), C. R. Nappi and A. Pasquinucci, Mod. Phys. Lett. A7, 3337 (1992).

4 G. W. Gibbons and M. J. Perry, Proc. R. Soc. London A358, 467 (1978), D. Page, Black Hole Physics, V De Sabbate and Z. Zhang, eds. 185 (1992), D. Hochberg, T. W. Kephart and J. W. York, Jr., Preprint IFP-467-UNC.

5 J. W. York, Jr., Phys. Rev. D28, 2929 (1983).

6 G. W. Gibbons, Commun. Math. Phys. 44, 245 (1975).

7 I. M. Ternov, A. B. Gaina and G. A. Chizov, Sov. J. Nucl Phys. 44, 343 (1986).

8 D. N. Page, Phys. Rev. D16, 2402 (1977).

9 W. A. Hiscock and L. D. Weems, Phys. Rev. D41, 1142 (1990).

10 Y. Kaminaga, Class. Quantum Grav. 7, 1135 (1990).

11 J. D. Beckenstein, Phys. Rev. D. 12, 3077 (1975).

12 I. Langmuir and L. Tonks, Phys. Rev. 34, 876 (1929), W. B. Thompson, An Introduction to Plasma Physics, (Addison-Wesley, Reading, MA) 1962. 
13 H. B. Callen and T. A. Welton, Phys. Rev. 83, 34 (1951).

14 D. Pavon and J. Rubi, Phys. Rev. D37, 2052 (1988).

15 D. Pavon, Phys. Rev. D43, 2495 (1991).

16 M. Morikawa, Phys. Rev. D 33, 3607 (1986).

17 E. Calzetta and B. L. Hu, Phys. Rev. D40, 656 (1989).

18 P. Candelas and D. W. Sciama, Phys. Rev. Lett. 38 ,1372 (1977).

19 E. Mottola, Phys. Rev. 33, 2136 (1986), Preprint LA-UR-92-1238.

20 J. S. Schwinger, Phys. Rev. 82, 664 (1951).

21 R. Kubo, J. Phys. Soc. Japan 12, 570 (1957), G. D. Mahan, Many Particle Physics, (Plenum, New York) 1990.

22 P. C. Martin and J. Schwinger, Phys. Rev.115, 1342 (1959).

23 C. Itzykson and J.-B. Zuber, Quantum Field Theories, (McGraw-Hill, New York) 1980;

S. Trieman, R. Jackiw and D. Gross, Lectures on Current Algebras and Their Applications,(Princeton University Press, Princeton, N.J.) 1972.

24 G. W. Gibbons and M. J. Perry, Phys. Rev. Lett. 36 , 985 (1976).

25 A. O. Caldiera and A. J. Leggett, Ann. Phys. (N.Y.) 149, 374 (1983).

26 K. S. Thorne, R. H. Price and D. A. McDonald, Black Holes: The Membrane Paradigm, (Yale University Press, New Haven, Conn.) 1986.

27 L. Susskind, L. Thorlacius and J. Uglum, hepth/9306069. 